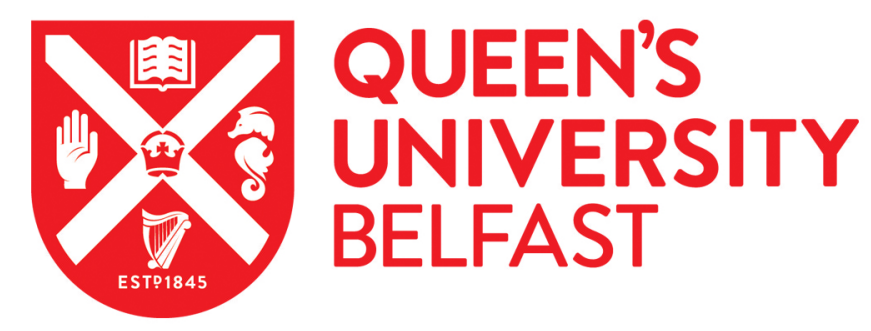

\title{
Review of Machine Vision-Based Electronic Travel Aids
}

Fei, Z., Yang, E., Hu, H., \& Zhou, H. (2017). Review of Machine Vision-Based Electronic Travel Aids. In Proceedings of the 23rd International Conference on Automation \& Computing, University of Huddersfield, Huddersfield, UK, 7-8 September 2017

\section{Published in:}

Proceedings of the 23rd International Conference on Automation \& Computing, University of Huddersfield, Huddersfield, UK, 7-8 September 2017

\section{Document Version:}

Peer reviewed version

Queen's University Belfast - Research Portal:

Link to publication record in Queen's University Belfast Research Portal

\section{Publisher rights}

(c) 2017 CACS.

This work is made available online in accordance with the publisher's policies. Please refer to any applicable terms of use of the publisher.

\section{General rights}

Copyright for the publications made accessible via the Queen's University Belfast Research Portal is retained by the author(s) and / or other copyright owners and it is a condition of accessing these publications that users recognise and abide by the legal requirements associated with these rights.

Take down policy

The Research Portal is Queen's institutional repository that provides access to Queen's research output. Every effort has been made to ensure that content in the Research Portal does not infringe any person's rights, or applicable UK laws. If you discover content in the Research Portal that you believe breaches copyright or violates any law, please contact openaccess@qub.ac.uk. 


\title{
Review of Machine Vision-Based Electronic Travel Aids
}

\author{
Zixiang $\mathrm{Fei}^{1}$, Erfu Yang ${ }^{1}$, Huoheng $\mathrm{Hu}^{2}$, Huiyu $\mathrm{Zhou}^{3}$ \\ ${ }^{1}$ Department of Design, Manufacture and Engineering Management, \\ University of Strathclyde, Glasgow G1 1XJ, Scotland, UK \\ ${ }^{2}$ School of Computer Science and Electronic Engineering \\ University of Essex, Colchester CO4 3SQ, \\ England, UK \\ ${ }^{3}$ School of Electronics, Electrical Engineering and Computer Science, \\ Queen's University Belfast, Belfast BT7 1NN, \\ Northern Ireland, UK
}

\begin{abstract}
Visual impaired people have navigation and mobility problems on roads. Up to now, many approaches have been proposed to help these people navigate around using different sensing techniques. This paper reviews several machine vision-based Electronic Travel Aids (ETAs) and compares them with those using other sensing techniques. The functionalities of machine vision-based ETAs are categorized from low-level image processing such as detection of the road regions and obstacles to high-level functionalities such as recognition of digital tags and texts. In addition, the characteristics of the ETA systems for blind people are discussed.
\end{abstract}

Keywords-Machine Vision, Visusal impairment, Navigation System, Electronic Travel Aids

\section{INTRODUCTION}

According to the statistics of the World Health Organization (WHO), there are approximately 285 million people who are visually impaired worldwide [1] [2]. In fact, 39 million people are blind and 246 million people have low vision. Most of the visually impaired people live in restricted circumstances and most of the blind people are 50 years old or over. Blind people suffer from many mobility problems such as avoiding obstacles, locating position, finding a pathway and accessing information [3].

Walking canes and guide dogs are the most common navigation assistants nowadays [4]. A cane is used for obstacle detection and a dog is good at obstacle avoidance [5]. However, they both have their own disadvantages. Very little navigation information can be provided by a cane because of the short range [6]. On the other hand, although a guide dog does well in navigation guide for blind people, very few patients can afford it [6] [7]. Both aids cannot communicate with blind people using natural ways such as speech. The blind people can only follow the guide dogs passively, or walk slowly using canes.

With the recent development of science and technology, more and more Electronic Travel Aids (ETAs) have been developed to help visual impaired people. Electronic Travel Aids are the devices that can convert the information obtained by vision and other sensors into the information that can be heard or touched by blind people [8] [9]. A typical ETA system could be divided into four parts: sensors as input, position beacons, information processing and user interface and output, which are shown on Fig. 1.

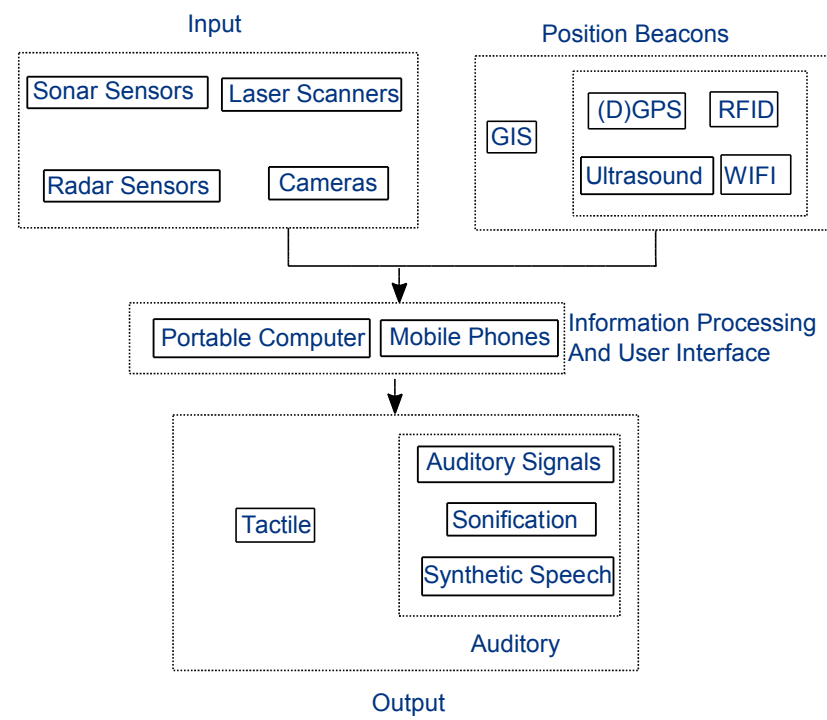

Figure 1 Illustration of a typical ETA System.

In an ETA system the first part is related to different sensors. The ETA can gather navigation information using sonar sensors, laser scanners or cameras [8]. Sonar-based navigation systems cannot provide precise information such as shape and velocity of objects, but machine visionbased systems can capture the whole image of the environment [6]. Therefore, machine vision technologies could help blind users in path finding, object identification, text extraction and information acquisition [10].

The second part in an ETA system is position beacons. RFID and WIFI are normally used in indoor environments, while GPS is used outdoors. GIS can be combined with positioning technologies to find the best ways to walk towards the destination. For instance, Ran proposed a system called 'Drishti' which uses Differential Global Positioning System (DGPS) outdoors and ultrasound sensors in indoor situations [11]. However, some alternative methods can be used to locate positions better. For example, Treuillet [12] only used machine 
vision to locate positions along paths which had already been learned and stored in the database.

The third part in the ETA system conducts information processing and user interaction. In order to improve the effectiveness of informing blind users of navigation information, a large amount of research work has been conducted. The users can be informed with the road situation so that they can decide if going straight ahead or turning left.

The final part of an ETA system is the system output. There are two kinds of outputs, namely auditory and tactile. In the survey [17], auditory assistive devices are classified into two categories: (i) the ETAs that can use binary auditory signals to aid a blind user, such as a warning tone; (ii) the ETAs that can use synthetic speech to aid the blind such as an optimal way towards the destination. On the other hand, tactile technologies have been widely deployed to investigate the size and position of an obstacle and road situations, such as the project of on-shoe tactile display and the project of tactile belt [13] [14]. In addition, a 2D Vibration Array and a 2D Tactile Vocabulary were deployed to improve the technology of Tactile-Visual Sensory Substitution [15] [16]. On the other hand, some ETAs can encode sound information. For example, the Stereo Vision based Electronic Travel Aid (SVETA) could transform stereo images into stereo sounds to inform the user such as the distance and size information of obstacles on roads [18].

There are also some relevant survey papers about ETA systems. Ruxandra et al reviewed 12 ETA systems in the aspect of the functions, advantages and limitations of each system and also offered a review about the capabilities of each ETA system such as wearability, portability and reliability [9]. Karen et al reviewed some technologies to assist blind people in navigation and shopping. Also, they concluded that RFID is more suitable for pedestrian navigation than GPS. In addition, barcode and QR code identification may be used for products identification [48]. Pawel reviewed the ETA systems in major mobility tasks such as orientation, obstacle avoidance and navigation and discussed their advantages and limitations [31]. It is clear that the previous research work has been mainly focused on pathway finding, obstacle avoiding and outdoor navigation [19]. However, with the help of machine vision, a navigation system can tell blind users where they are, location of certain items, road situation, ways to the destination and texts information on roads. The system can be portable and communicate with the blind people in sounds. This technology can also play an important role in robot navigation, when they need to move and know about location information. This paper is focused on the Electronic Travel Aids using machine vision. The content covered in the paper is shown in Fig. 2.

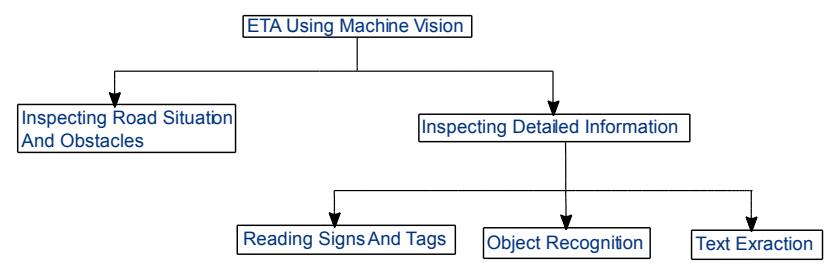

Figure 2 Review Contexts.
The rest of the paper is organized as follows. After a brief introduction in Section 1, Sections 2 explains how to inspect road situation and obstacles. Section 3 introduces Digital Signs, Color Markers or RFID Tags used in ETAs. In Section 4, text tracking and recognition technologies in ETAs are briefly described. Section 5 presents some object detection and recognition methods for guiding blind people. Finally, a brief summary is given in Section 6 .

\section{INSPECTING ROAD SITUATION AND OBSTACLES}

Blind people may not be able to prevent some dangerous situations such as obstacles or holes on roads, even when they walk alone with a cane. To detect obstacles, many kinds of sensors can be used, such as sonar, radar, infrared Intensity, laser and infrared Flight of Time [20]. However, all these methods have their own problems and conditions. For example, sonar ranging sensors have several potential errors such as Specularity in calculating ranges. With regards to radar, although the working range is long, the system using radar has blind spots in a large vertical angular range. Furthermore, a laser scanner has advantages in a long range, high accuracy and speed, but suffers from expensive costs and narrow ranges. On the other hand, navigation systems based on machine vision seem to be more powerful and flexible than others. In general, vision based navigation systems can provide blind people with navigation information such as path planning and obstacle avoidance on roads [22] [49].

\section{A. For Normal Blind Users}

Balakrishnan et al. proposed a Stereo Vision based Electronic Travel Aid (SVETA) system that was comprised of stereo earphones, stereo cameras and a computing device [21]. The system can guide blind people by informing them with the information of obstacles such as shape, size, position and distance. The time for the system to process an image and give feedback is about 1.25 seconds. However, the system may have some errors when lighting is strong. With the help of this navigation system, blind people can know the road situation clearly by listening to the stereo musical sound from the system. The disadvantage is that blind people need training to understand the stereo musical sound produced by the SVETA system.

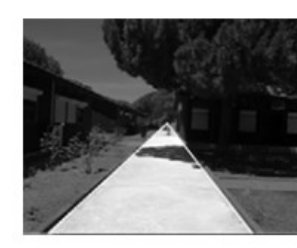

(a) Detecting the path

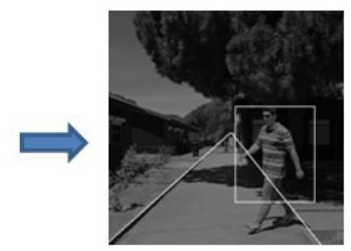

(b) Detecting the path and the obstacle
Figure 3 Detecting the path and obstacle in SmartVision [22].

Jose et al. proposed a SmartVision navigation aid for blind people [22]. The SmartVision system focuses on the local navigation for a blind user in two aspects: detecting a path and obstacles. The whole system is mainly made up of the following components: the stereo camera attached 
at the height of the chest, the portable computer which can be worn on the shoulder or in the pocket and one earphone which can be replaced by a speaker. As shown in Fig. 3, the border of the path is detected and then the path region is highlighted. Also, the obstacle is detected. The SmartVision system which works at a few frames per second can also detect static and moving objects and will alert the user if an object is detected.

Shang et al. also proposed a navigation system which is based on machine vision to guide blind people [23]. This system can provide information about road regions and help blind people avoiding stationery and moving objects. Experiments show that the system can work in real time and has a frame rate of 12 frames per second. The system is mainly focused on finding a road region, but cannot provide the user with the information such as the velocity of a moving object.

Jie et al. proposed a novel electronic travel aid for blind people which can be used to detect obstacles, recognize traffic lights and detect road edges [24]. The system consisted of two cameras, a portable computer and an earphone. The experiment of the system using 75 consecutive frames shows that the recognition rate is $96 \%$ and the processing speed is about $0.08 \mathrm{~s}$ per frame. The proposed method is to compare gray values by converting a color image into the gray scale. However, if the obstacle has similar gray values to those of non-obstacles, the obstacle cannot be detected.

\section{B. For Blind Users Who Need Wheelchair}

About one in ten blind people use wheelchairs [25], [50]. For blind wheelchair users, independent travel is challenging and using a cane or a guide dog is impractical. ETAs may play a significant role in this situation. ETA equipments do not need to be portable, for they can be equipped on a wheelchair.

Coughlan et al. performed a research project to sense terrain features including curbs, drop-offs and shoreline for wheelchair navigation with the help of computer vision [25]. The cameras situated on the wheelchair can take images and the developed vision algorithms can process the visual information in real time. The audio and tactile feedback help the user for navigation and control the wheelchair. The ground plane in the scene is estimated first and the range map is converted to an elevation map in order to find the terrain features including drop-offs and curbs. However, the performance of the elevation map can be influenced by a relatively small elevation discontinuity.

Ivanchenko et al. also proposed a system to alert a blind user with the terrain information, who needs a wheelchair to support [26]. The system aims to extend the reach of the walking cane. In the system, the user can recognize the environment by sweeping a cane with the help of computer vision technologies. Moreover, the system will alert the user in sound if a terrain feature (such as an obstacle, wall or curb) is detected in the direction that the cane is pointing. This method may be limited in terms of the working area.

Nguyen proposed a semi-autonomous wheelchair system named as TIM (Thought-controlled Intelligent
Machine), which uses stereoscopic vision for 3D depth perception along with a spherical camera for 360-degrees of monocular vision [27]. The vision system can help the user avoid the collisions with other people or obstacles during the autonomous navigation. The experiment is conducted in crowded places. The experiment shows a good performance in obstacle avoiding with the help of machine vision technology.

\section{USING DigITAL SIGNS, COLOR MARKERS OR RFID TAGS}

To help blind people, many researchers are working towards an optimal solution for scanning or recognition barcode, color markers or RFID information grid which includes the information of locations, descriptions of navigation and wayfinding [28] [29]. These special markers can work as landmarks and signs in the navigation process.

Both digital tags using machine vision and RFID tags have their own problems and difficulties. Computer vision will make it cost effective [30]. However, computer vision techniques may suffer from motion blur, image quality or illumination change. On the other side, RFID tags may have a weakness in their working range. According to Karen Duarte, active tags need external power supply although they cover a larger range [31].

\section{A. Using RFID}

Willis and Helal proposed a RFID based navigation system which contains location information and related descriptive information [32]. It is of low cost and is easy for installation and maintenance. One possible practical usage for this system is to help navigation of blind students in college buildings where corridors, hallways and rooms are facilitated with RFID tags. In this system, a blind user will be able to navigate by scanning RFID tags at the place with a RFID reader attached to the shoe or the cane. Each tag can store information about 2000 bits, and the time for reading the tags is short enough and will not influence the walking. However, in order to locate direction, 2 or more tags need to be detected. In addition, in order to read the tags, the distance between the reader and the tags needs to be less than $100 \mathrm{~mm}$ unless a large antenna and strong RF signals are used.

The layout of the installation of the RFID tags in a building is also important. Tsirmpas created the scenarios with the installation of RFID tags to test if the system could help blind people travel in an unknown place [28]. Experiments show that the success rate of avoiding obstacles achieved by the system is about $99 \%$.

Szeto researched on extending the range of RFID tags [29]. They have ideas such as creating active RFID tags which use solar power and will not need any battery. Furthermore, by installing multiple directional antennas on a jacket, scanning the environment will be no longer needed. However, this work still seems to be in a research stage.

\section{B. Using Machine Vison}

Although there is a significant development in RFID technology, its major weak point is the short distance 
between the reader and the passive RFID tags. Tjan et al. proposed an indoor navigation system using inexpensive digital signs for vision impaired people providing location, heading and other information within a distance of 3 meters [33]. The digital tags can be found by using a hand-hold device called Magic Flashlight which contains an array of infrared emitters. The Magic Flashlight uses a camera to identify a tag when the tag is in the field of view. The tag is slightly larger than a credit card and contains a 16-bit number which provides navigation information by checking the 16-bit number in the database.

Machine vision is used to translate the pattern on the retro-reflective tag to a 16-bit number [33]. Reading digital tags using machine vision is a better way than detecting the RFID signals in terms of working distance. However, the tag identification process will be affected by sunlight. Furthermore, experiments show that the tags identification takes about 20 seconds which is difficult for real time navigation, although it can be improved.

Different blind people may have different navigation behaviors [5]. Manduchi et al. presented a usability study of a navigation system for blind people by detecting color markers [3]. In the study, a normal mobile phone's onboard camera was used to detect some color makers placed in the environment in order to locate their positions. There will be a beeping sound to inform the user if the color makers are detected. In addition, the beeping sound repeats more frequently if the distance between the color maker and the mobile phone is less than 4.5 meters. Three blind participants took part in the test in different indoor places. Only one participant succeeded in all the tests. The experiment showed that the field of view is an important factor in this navigation process. It was clear that some navigation technology may not meet all the navigation behaviors.

In summary, RFID tags or digital signs can help blind people recognize objects or landmarks and be alternative methods to provide position and heading information. However, compared with the GPS location technology in outdoor conditions and location technology using a WIFI technology which may be less accurate, RFID tags or digital signs based system may be more expensive.

\section{TEXT TRACKING AND RECOGNITION}

Without the help of text information when traveling, important information will be missed such as the name of roads and important signs. In order to inform blind people of text information when traveling, the system needs to be small enough to take or wear, whilst it can recognize the text information in real time and use an appropriate way to inform the user. In practice, researchers work on extracting text information from the environment and converting it into a form that most off-shelf OCR systems can process. In general, there are two kinds of problems: (i) selecting good frames that are unblurred and containing text information from videos and (ii) extracting text information that most OCR systems can process from image frames.

\section{A. Text Extraction}

Tian et al. developed a text extraction and recognition algorithm in a navigation system for blind people [34] [35]. The system can extract text regions from signs in complex backgrounds and then use topological analysis to filter out the interference. The text information from a sign can be used as landmarks in the global navigation. They conducted experiments by selecting 420 images containing compatible text information and achieved the accuracy of about $71 \%$. However, there are some limitations in the system. For instance, if the color of the background is similar to that of the text, the interference cannot be filtered out. This algorithm also fails if the text information is in different colors or the texts are less than 3 letters.

Most off-shelf OCR products cannot recognize texts which are skew or slant severely. Hairuman proposed an improved OCR signage recognition model for blind people to recognize a text which is skew or slant [36]. If the text is skew, the Shearing Transformation will be applied. The model is tested using 15 different samples of texts from 5 subjects and achieved a recognition rate of $82.7 \%$.

$\mathrm{Yi}$ et al. developed a prototype system to read text information on an object which can be held in hand by blind people [37]. The object of interest is located by blind people using a motion-based segmentation method. To extract the text information from the background containing colorful noise, a text localization algorithm was proposed by using the stroke orientations and edge distributions. To enable the system to focus on an object of interest in a complicated environment, the user needs to shake the object. Fig. 4 shows the flowchart of reading text information from objects for blind users. In addition, the experiments showed that the method had low accuracy and took about 2 seconds to process every image frame.

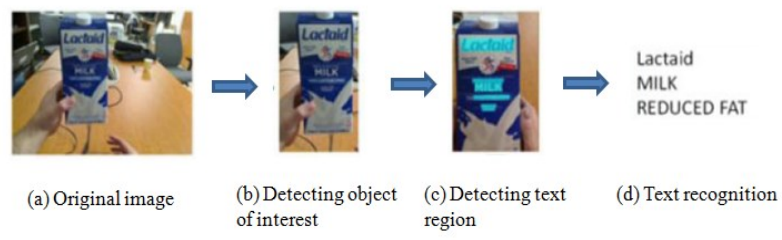

Figure 4 Flowchart of Reading Text Information from Objects for Blind Users [37]

Public transportation systems play an important role in people's traveling from one place to another. However, it is difficult for vision impaired people to notice the coming buses and recognize route numbers of buses. Pan et al. [38] proposed a primary framework to detect and recognize a bus route number using the images taken from a camera and notify the user in speech. The image containing bus numbers is captured by a blind user's device. The text region is located and the non-text region is removed by using the layout analysis and applying a text classifier. The experiment shows that only part of the text regions are detected using the bus images containing 438 text regions. This algorithm relies on the layout of a bus. 
Karacst also invented a Bionic Eyeglass using machine vision to guide blind people to recognize a bus number [39]. The system can inform blind people with the bus number by detecting the bus number from the recorded videos. A video is recorded at least 15 frames per second. They have tested the Bionic Eyeglass using 35 videos with lengths of 15 to 90 seconds, achieving a recognition rate of about $83 \%$.

\section{B. Selecting the Appropriate Images for Text Extracting}

Most researchers prefer to use off-shelf OCR products in their systems. In general, a navigation system using OCR for blind people consist of three parts: extracting text information, using OCR products to recognize texts and informing the user of the text information in voice. In practice, extracting text information can be divided into two steps: to acquire an image of a small area of the object of interest from a video and to extract the text information in images. Most of researchers work on the second part by designing an algorithm to extract texts from complex background. However, in real world an advanced and qualified OCR system for blind people needs to be able to extract the text information from videos in real time. It will involve selecting appropriate images for text extraction from videos.

Tian proposed a method to detect unblurred frames in a video captured by blind users [40]. Four types of features which are based on edges and luminance information are used to check if an image frame is blurred. In order to evaluate the method, 17 videos were captured both indoors and outdoors when the user was moving. The accuracy is $84.1 \%$.

Goto and Tanaka proposed a prototype system which was equipped with a video camera to extract text information from natural scenes in real time [41] [42]. They noticed that the movement of the user is usually unpredictable and a robust text tracking method is needed. Meanwhile, some text information may appear in consecutive image frames. Recognition of the text information in all images is unnecessary and impractical. Furthermore, the user may want to know the text information at a specific moment. In practice, the text regions were extracted using a revised discrete cosine transformation (DCT) based method. In addition, similar text regions were grouped into images chains using a particle filter based method and the best image is selected from each image chain in order to avoid recognizing repeated text information.

In summary, the system showed above succeeded in detecting and tracking the text region in video sequences, grouping image frames containing the same text information and selecting proper text images which can be used for text recognition [41] [42]. They have tested their system using 1730 image frames and reduced the images of text candidate regions to $1.47 \%$ at the rate of about 10 fps.

\section{OBJECT DETECTION AND RECOGNITION}

When visual impaired people walk around, a navigation system can be used to detect landmarks or amenities such as door and elevator. Recognizing objects by comparing two images using image features may be effective for identifying part of an object.

\section{A. 3D Template Match}

Tian et al. proposed a wayfinding aid for blind people using computer vision to find landmarks and some building amenities such as an elevator or an exit [34] [35]. They proposed an algorithm to detect doors by using a general door model containing features such as edges and corners of a door. The experiment with 221 door images showed that the algorithm was robust to various light conditions, sizes, color, text information, viewpoints and occlusions and achieved a successful rate of about $90 \%$. Using the edge and text information to detect a door is shown on Fig. 5.
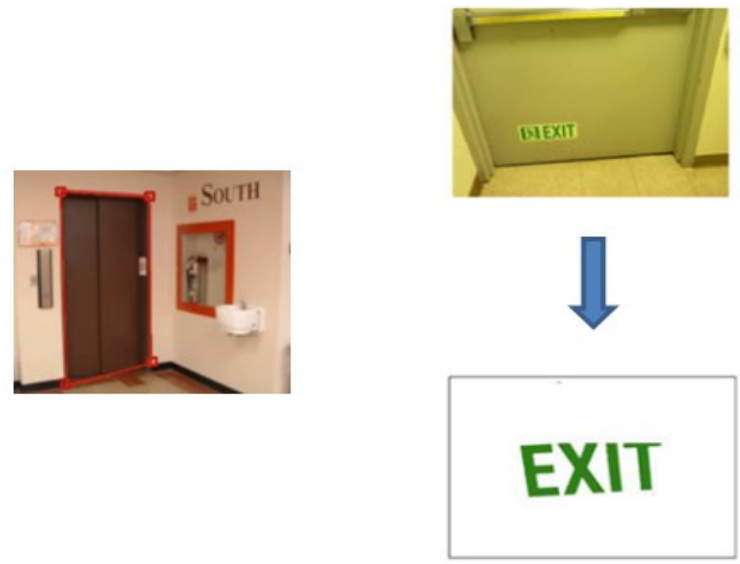

$$
\begin{aligned}
& \text { (a) Detecting door } \\
& \text { edges }
\end{aligned}
$$

(b) Detecting text on the door

Figure 5 Using edge and text information to detect a door [34].

Serrao et al. presented a system using a camera to find landmarks and localize the user [4] [43] [44]. The system was integrated with an indoor GIS which includes landmarks and objects in order that it helps trace and validate a route for the user's navigation. The open SURF (Speeded-up Robust Features) library was used. The system can operate in real time by both the sighted and blind people and show the good results in a range of about five meters. The system performance deteriorates in severe background noise and light changing.

Wang proposed a framework to detect stairs and pedestrian crosswalks which are normally comprised of parallel lines using a RGBD camera which includes depth information [45]. The experiment shows that the proposed method can achieve a recognition rate of about $90 \%$ for detecting stairs and pedestrian crosswalks and the system can work in real time. However, some items which contain parallel lines such as bookshelves could be misclassified as stairs and pedestrian crosswalks. Moreover, the depth information is hard to obtain when lighting changes.

The attempts shown above are based on machine vision and are used for blind people to recognize objects regardless of size, color, position and so on. However, they have some weaknesses. The recognition performance could be influenced by motion blur, light condition and 
image quality. A lot of work needs to be done to build the 3D templates of objects and make these algorithms be computationally demanding.

\section{B. 2D Image Matching}

Matusiak et al. proposed a software module for blind people to recognize objects which can run on an Android smartphone [46]. This software can recognize objects by matching the images captured by the mobile phones against a database of objects. In order to recognize an object better, the Scale-Invariant Feature Transform (SIFT) computer vision algorithm was applied. In addition, this software was also able to detect the major color of the image and locate the brightest place. The experiment showed that the accuracy of recognizing the front side of 30 products was $90 \%$. Although the object recognition performance was promising, this algorithm can only recognize objects in 2D instead of 3D. Moreover, the average time for object comparison was about 30 seconds. Certainly, the performance of the system would also be affected by the quality of the images and light condition.

\section{Using Other Sensors}

Object recognition could also be conducted by using other methods. Scherlen proposed a new concept named RecognizeCane which can be used to recognize some common objects [47]. RecognizeCane used brilliance, infrared or water sensors to detect subjects such as a water puddle or a zebra crossing. In order to detect several subjects, several sensors may be needed. For instance, a water detector is fitted at the end of a cane to detect water puddle, and a brilliance sensor equipped with an infrared transmitter and a receiver is used to detect zebra crossing as it can differentiate a white surface from a black one.

\section{CONCLUSIONS}

This paper has reviewed several Electronic Travel Aids, especially for helping blind people navigation using machine vision technologies. It classifies the navigation functionalities provided by vision-based ETAs from lowlevel image processing such as detecting road regions and avoiding obstacles to high- level functionalities such as navigation using digital tags and recognizing object or text.

With the continuous development of sensor technologies, vision-based ETAs will have significant improvement in the near future. It is clear that visionbased navigation systems can provide blind users with the navigation information such as location, the best way to the destination, road situation, important landmarks, items and text information. In addition, it will also be portable, reliable, real time, low cost, and robust. Finally, it could be used to aid sighted people or even robots such as searching specific items in complex environments and informing users of text information on a distant notice board.

\section{ACKNOWLEDGMENTS}

Z. Fei and E. Yang are supported in part by Strathclyde's Strategic Technology Partnership (STP) Programme with CAPITA (2016-2019). The authors thank Dr Neil Mackin (CAPITA mentor) and Miss Angela Anderson (the STP's coordinator) for their support. The contents including any opinions and conclusions made in this paper are those of the authors alone. They do not necessarily represent the views of CAPITA plc. H. Zhou is supported by UK EPSRC under Grants EP/N508664/1, EP/R007187/1 and EP/N011074/1, and Royal SocietyNewton Advanced Fellowship under Grant NA160342.

\section{REFERENCES}

[1] who.int. (2014, August). Visual impairment and blindness [online]. Available: http://www.who.int/mediacentre/factsheets/fs282/en/

[2] who.int. (n.d.). Global data on visual impairment [online]. Available: http://www.who.int/blindness/publications/globaldata/en/

[3] R. Manduchi, S. Kurniawan, and H. Bagherinia, "Blind guidance using mobile computer vision: A usability study," in ASSETS'10 Proceedings of the 12th International ACM SIGACCESS Conference on Computers and Accessibility, 2010.

[4] M. Serrão et al., "Computer vision and GIS for the navigation of blind persons in buildings," Univers. Access Inf. Soc., vol. 14, no. $1,2015$.

[5] M. A. Williams, A. Hurst, and S. K. Kane, "Pray before you step out': Describing personal and situational blind navigation behaviors," in Proceedings of the 15th International ACM SIGACCESS Conference on Computers and Accessibility, ASSETS 2013, 2013.

[6] J. Liu, J. Liu, L. Xu, and W. Jin, "Electronic travel aids for the blind based on sensory substitution," in ICCSE 2010 - 5th International Conference on Computer Science and Education, Final Program and Book of Abstracts, 2010.

[7] S. Shao, MOBILITY AIDS FOR THE BLIND. 1985

[8] D. Dakopoulos and N. G. Bourbakis, "Wearable obstacle avoidance electronic travel aids for blind: A survey," IEEE Trans. Syst. Man Cybern. Part C Appl. Rev., vol. 40, no. 1, 2010.

[9] R. Tapu, B. Mocanu, and E. Tapu, "A survey on wearable devices used to assist the visual impaired user navigation in outdoor environments," in 2014 11th International Symposium on Electronics and Telecommunications, ISETC 2014 - Conference Proceedings, 2015.

[10] A. Sadat Mohammed Yasin, M. Majharul Haque, S. Binte Anwar, and M. Shakil Ahamed Shohag, "Computer Vision Techniques for Supporting Blind or Vision Impaired People: An Overview," Int. J. Sci. Res. Eng. Technol., vol. 2, no. 8, pp. 498-503, 2013.

[11] L. Ran, S. Helal, and S. Moore, "Drishti: An integrated indoor/outdoor blind navigation system and service," in Proceedings - Second IEEE Annual Conference on Pervasive Computing and Communications, PerCom, 2004.

[12] S. Treuillet, E. Royer, T. Chateau, M. Dhome, and J.-M. Lavest, "Body mounted vision system for visually impaired outdoor and indoor wayfinding assistance," in CEUR Workshop Proceedings, 2008, vol. 415.

[13] L. A. Johnson and C. M. Higgins, "A navigation aid for the blind using tactile-visual sensory substitution," in Annual International Conference of the IEEE Engineering in Medicine and Biology Proceedings, 2006.

[14] R. Velázquez and O. Bazán, "Preliminary evaluation of podotactile feedback in sighted and blind users," in 2010 Annua International Conference of the IEEE Engineering in Medicine and Biology Society, EMBC'10, 2010.

[15] D. Dakopoulos, S. K. Boddhu, and N. Bourbakis, "A 2D vibration array as an assistive device for visually impaired," in Proceedings of the 7th IEEE International Conference on Bioinformatics and Bioengineering, BIBE, 2007.

[16] D. Dakopoulos and N. Bourbakis, "Towards a 2D tactile vocabulary for navigation of blind and visually impaired," in 
Conference Proceedings - IEEE International Conference on Systems, Man and Cybernetics, 2009.

[17] R. W. Massof, "Auditory assistive devices for the blind," 2003.

[18] G. Balakrishnan, G. Sainarayanan, R. Nagarajan, and S. Yaacob, "Stereo image to stereo sound methods for vision based ETA," in 2005 1st International Conference on Computers, Communications and Signal Processing with Special Track on Biomedical Engineering, CCSP 2005, 2005.

[19] S. R. A. W. Alwi and M. N. Ahmad, "Survey on outdoor navigation system needs for blind people," in Proceeding - 2013 IEEE Student Conference on Research and Development, SCOReD 2013, 2015.

[20] S. Lalar, "Obstacle Detection Sensors: A Survey," vol. 3, no. 5, 2013.

[21] G. Balakrishnan, G. Sainarayanan, R. Nagarajan, and S. Yaacob, "Wearable Real-Time Stereo Vision for the Visually Impaired."

[22] J. José, M. Farrajota, J. M. Rodrigues, and J. M. Hans du Buf, "The smart vision local navigation aid for blind and visually impaired persons," Int. J. Digit. Content Technol. its Appl., vol. 5, no. 5,2011

[23] W. Shang, W. Jiang, and J. Chu, "A machine vision based navigation system for the blind," in Proceedings - 2011 IEEE International Conference on Computer Science and Automation Engineering, CSAE 2011, 2011, vol. 3

[24] Y. Jie and S. Yanbin, "Obstacle detection of a novel travel aid for visual impaired people," in Proceedings of the 2012 4th International Conference on Intelligent Human-Machine Systems and Cybernetics, IHMSC 2012, 2012, vol. 2.

[25] J. Coughlan, R. Manduchi, and H. Shen, Computer vision-based terrain sensors for blind wheelchair users, vol. 4061 LNCS. 2006.

[26] V. Ivanchenko, J. Coughlan, W. Gerrey, and H. Shen, "Computer vision-based clear path guidance for blind wheelchair users," in ASSETS'08: The 10th International ACM SIGACCESS Conference on Computers and Accessibility, 2008.

[27] J. S. Nguyen, T. N. Nguyen, Y. Tran, S. W. Su, A. Craig, and H. T. Nguyen, "Real-time performance of a hands-free semiautonomous wheelchair system using a combination of stereoscopic and spherical vision," in Proceedings of the Annual International Conference of the IEEE Engineering in Medicine and Biology Society, EMBS, 2012.

[28] C. Tsirmpas, A. Rompas, O. Fokou, and D. Koutsouris, "An indoor navigation system for visually impaired and elderly people based on Radio Frequency Identification (RFID)," Inf. Sci. (Ny)., vol. 320, 2015.

[29] A. Y. J. Szeto and S. K. Sharma, "RFID based indoor navigational aid for persons with severe visual impairments," in Annual International Conference of the IEEE Engineering in Medicine and Biology - Proceedings, 2007.

[30] N. Amin and M. Borschbach, "Classification criteria for local navigation digital assistance techniques for the visually impaired," in 2014 13th International Conference on Control Automation Robotics and Vision, ICARCV 2014, 1997.

[31] K. Duarte, J. Cecilio, and P. Furtado, "Overview of assistive technologies for the blind: Navigation and shopping," in 2014 13th International Conference on Control Automation Robotics and Vision, ICARCV 2014, 2014.

[32] S. Willis and S. Helal, "RFID information grid for blind navigation and wayfinding," in Proceedings - International Symposium on Wearable Computers, ISWC, 2005, vol. 2005.

[33] B. S. Tjan, P. J. Beckmann, R. Roy, N. Giudice, and G. E. Legge, "Digital Sign System for Indoor Wayfinding for the Visually Impaired."
[34] Y. Tian, X. Yang, C. Yi, and A. Arditi, "Toward a computer vision-based wayfinding aid for blind persons to access unfamiliar indoor environments," Mach. Vis. Appl., vol. 24, no. 3, 2013.

[35] Y. Tian, C. Yi, and A. Arditi, Improving computer vision-based indoor wayfinding for blind persons with context information, vol. 6180 LNCS, no. PART 2. 2010.

[36] I. F. B. Hairuman and O.-M. Foong, "OCR signage recognition with skew \&amp; slant correction for visually impaired people," in Proceedings of the 2011 11th International Conference on Hybrid Intelligent Systems, HIS 2011, 2011.

[37] C. Yi, Y. Tian, and A. Arditi, "Portable camera-based assistive text and product label reading from hand-held objects for blind persons," IEEE/ASME Trans. Mechatronics, vol. 19, no. 3, 2014.

[38] H. Pan, C. Yi, and Y. Tian, "A primary travelling assistant system of bus detection and recognition for visually impaired people," in Electronic Proceedings of the 2013 IEEE International Conference on Multimedia and Expo Workshops, ICMEW 2013, 2013.

[39] K. Karacs and T. Roska, "Route number recognition of public transport vehicles via the bionic eyeglass," in Proceedings of the IEEE International Workshop on Cellular Neural Networks and their Applications, 2006

[40] L. Tian, Y. Tian, and C. Yi, "Detecting good quality frames in videos captured by a wearable camera for blind navigation," in Proceedings - 2013 IEEE International Conference on Bioinformatics and Biomedicine, IEEE BIBM 2013, 2013.

[41] H. Goto and M. Tanaka, "Text-tracking wearable camera system for the blind," in Proceedings of the International Conference on Document Analysis and Recognition, ICDAR, 2009.

[42] M. Tanaka and H. Goto, "Text-tracking wearable camera system for visually-impaired people," in Proceedings - International Conference on Pattern Recognition, 2008.

[43] M. Serrão, J. M. F. Rodrigues, J. I. Rodrigues, and J. M. H. Du Buf, "Indoor localization and navigation for blind persons using visual landmarks and a GIS," in Procedia Computer Science, 2012, vol. 14

[44] M. Serrão, J. M. F. Rodrigues, and J. M. H. Du Buf, "Navigation framework using visual landmarks and a GIS," in Procedia Computer Science, 2013, vol. 27.

[45] S. Wang and Y. Tian, "Detecting stairs and pedestrian crosswalks for the blind by RGBD camera," in Proceedings - 2012 IEEE International Conference on Bioinformatics and Biomedicine Workshops, BIBMW 2012, 2012.

[46] K. Matusiak, P. Skulimowski, and P. Strurnillo, "Object recognition in a mobile phone application for visually impaired users," in 2013 6th International Conference on Human System Interactions, HSI 2013, 2013.

[47] A. C. Scherlen, J. C. Dumas, B. Guedj, and A. Vignot, "'RecognizeCane': The new concept of a cane which recognizes the most common objects and safety clues.," Conf. Proc. IEEE Eng. Med. Biol. Soc., 2007.

[48] P. Strumillo, "Electronic interfaces aiding the visually impaired in environmental access, mobility and navigation," in 3rd International Conference on Human System Interaction, HSI'2010 - Conference Proceedings, 2010

[49] H. Zhou, A. Wallace and P. Green, "A multistage filtering technique to detect hazards on the ground plane", Pattern Recognition Letters, vol. 24, no. 9-10, pp. 1453-1461, 2003.

[50] E. Rechy-Ramirez and H. Hu, "A Flexible Bio-Signal Based HMI for Hands-Free Control of an Electric Powered Wheelchair", International Journal of Artificial Life Research, vol. 4, no. 1, pp. 59-76, 2014. 